\title{
AUTOEVALUACIÓN DE LAS INTELIGENCIAS MÚLTIPLES Y RENDIMIENTO ACADÉMICO EN ESTUDIANTES DE ADMINISTRACIÓN Y GERENCIA DE UNA UNIVERSIDAD DE LIMA
}

\author{
Natividad ESPÍRITU SALINAS, \\ Jorge CARBAJAL ARROYO, \\ William TORRES ACUÑA, \\ José Antonio CANALES SIERRALTA; y \\ Américo SOTO GRIMALDO \\ Universidad Ricardo Palma \\ natypsicologia@yahoo.es
}

\section{RESUMEN}

Se correlacionó las inteligencias múltiples con el rendimiento académico en 100 estudiantes de administración y gerencia de una universidad privada de Lima, Perú (48 varones y 52 mujeres), de 6 to al 10 mo semestre, de 20 a 35 años de edad. El instrumento fue el Cuestionario de Autoevaluación de las Inteligencias Múltiples (CAIM) del Dr. Roberto Kestész, que mide inteligencia Intrapersonal, Interpersonal,Verbal-Lingüística,Visual-Espacial, Lógico-Matemática, Musical-Rítmica y Corporal-Kinestésica. En rendimiento académico se encontró un promedio de notas histórico de 12.19, en sistema vigesimal. En inteligencias múltiples, de 0 a 100, se observó mayor puntaje en Inteligencia Intrapersonal (82.09) y menor puntaje en Inteligencia Musical - Rítmica (68.4). Se encontró correlación positiva (Pearson, $p<0.05$ ) entre el rendimiento académico y las inteligencias múltiples Intrapersonal, Interpersonal, Verbal-Lingüística, VisualEspacial y Lógico-Matemática, sin embargo esta relación no era estadísticamente significativa. Correlacionaron negativamente (estadísticamente no significativo) el rendimiento académico y las inteligencias Musical-Rítmica y Corporal-Kinestésica.

\section{PALABRAS CLAVE}

Inteligencias múltiples, Rendimiento académico, Estudiantes administración y gerencia

\section{SELF-EVALUATION OF MULTIPLE INTELLIGENCES AND ACADEMIC PERFORMANCE IN STUDENTS OF ADMINISTRATION AND MANAGEMENT OF A UNIVERSITY OF LIMA}

\section{ABSTRACT}

Correlated multiple intelligences with academic performance in 100 students of administration and management of a private University in Lima, Peru ( 48 males and 52 females), from 6th to 10th semester of 20 to 35 years of age. The instrument was the questionnaire of self-evaluation of the 
intelligences multiple (CAIM) of the Dr. Roberto Kestesz, measuring intelligence Intrapersonal, Interpersonal, Verbal - linguistics, spatial, Logical -mathematics, Musical - rhythmical and body - kinesthetic. Academic historical notes average of 12.19, found in twentieth. Multiple intelligences, from 0 to 100, showed higher score in Intrapersonal intelligence (82.09) and lowest score in Musical intelligence - rhythmic (68.4). Positive correlation was found (Pearson, $p$ \&lt; 0.05 ) between academic performance and multiple intelligences Intrapersonal, Interpersonal, Verbal -linguistics, spatial and Logical - mathematics, but this relationship was not statistically significant. They correlated negatively (statistically nonsignificant) academic performance and the Musical - rhythmical intelligences and body-kinesthetic.

\section{KEYWORDS}

Multiple Intelligences, Academic Performance, Students administration and management

Recibido: $12 / 6 / 2017$

Aprobado: $30 / 11 / 2017$ 


\section{Introducción}

F

errándiz, Prieto, Bermejo y Ferrando (2006) destacaron la utilidad del modelo de las Inteligencias Múltiples para diseñar perfiles cognitivos, especialmente para detectar alumnos excepcionales con puntos fuertes y lagunas o deficiencias y por lo tanto de utilidad para diseñar y ejecutar estrategias de atención a la diversidad, basadas en esta filosofía de las Inteligencias Múltiples. La conceptualización sobre las inteligencias múltiples en el ámbito educativo ofrece propuestas para lograr una educación de calidad, basadas en el desarrollo de las capacidades de los alumnos y la atención a la diversidad (Gonzales y López, 2006).

Howard Gardner (1983) propuso el concepto de inteligencias múltiples, considerándola no como un elemento fijo einmodificable, indisoluble de aptitudes lingüísticas, matemáticas y espaciales, sino como un modelo cognitivo donde participaban inteligencias musical, naturalista y corporal, además de elementos vinculados con el desarrollo emocional, como la inteligencia intra e interpersonal. Planteó la multidimensionalidad de la inteligencia como la capacidad para resolver problemas y crear productos valorados culturalmente. De esta manera, no existe una única manifestación de conducta inteligente sino que hay diversas formas en las que la inteligencia puede manifestarse (Carpintero, Cabezas y Sánchez, 2009). Las inteligencias planteadas por Gardner inicialmente fueron las 7 siguientes (Gardner, 2005; Sánchez, 2007; Cossio, 2007; Del Pozo, 2008):

Inteligencia lingüística.- Inteligencia más universal, de los escritores (narrativa, prosa, poesía, etc.), oradores, periodistas, etc. Implica habilidad para manejar el significado de las palabras de forma oral o escrita. Habilidad en uso de la sintáxis, fonética, semántica y usos pragmáticos del lenguaje (retórica, mnemónica, explicación y metalenguaje). Hábitos y conductas vinculadas con este tipo de inteligencia: Gusto por la lectura de libros y revistas, buena memoria para nombres, letras de poesías y canciones, habilidad para hablar, inventar y contar cuentos e historias, expresividad verbal con facilidad, gustan exponer oralmente, tienen habilidades e intereses por aprender nuevos términos o vocabulario e idiomas, jugar con rimas, trabalenguas.

Inteligencia lógico-matemática.- Inteligencia de los científicos, de los que les gusta investigar, conocer, explicar, que poseen un mayor juicio crítico, analizan con facilidad planteos y resuelven problemas, realizan cálculos numéricos, estadísticas, presupuestos, razonamientos lógicos. Fue la inteligencia explicada por Piaget, quien pensó que así estaba estudiando la inteligencia, pero realmente estaba estudiando más la inteligencia lógico matemática. Comprende el proceso de abstracción y exploración de problemas racionales difíciles de lograr. Implica rigurosidad del pensamiento formal y objetivo. Tienen como competencias básicas el razonar de forma deductiva e inductiva, relacionar conceptos, operar con conceptos abstractos como números que representen objetos concretos. Hábitos y conductas vinculados con este tipo de inteligencia: Facilidad para el cálculo numérico, gusto por juegos que implican pensar, gusto de saber el qué y el porqué de las cosas, por adivinanzas y acertijos. Las personas que tienen desarrollada esta inteligencia son observadores, analíticos y hacen muchas preguntas. 
Inteligencia musical.- Inteligencia que crea, interpreta y aprecia la música. Incluye la sensibilidad al ritmo, tono y timbre. Está relacionada con la imaginación auditiva. Se formula en representaciones sonoras musicales que se combinan con la experiencia emocional. Caracteriza a las personas capaces de producir composiciones musicales y que tienen habilidades para tocar instrumentos musicales. Hábitos y conductas vinculadas con este tipo de inteligencia: Gusto por escuchar música y seguir su compás, participar en el coro, tararear o cantar canciones, conocer y dominar algún instrumento musical, inventar o crear canciones. Está presente en compositores, directores de orquesta, críticos musicales, músicos y oyentes sensibles.

Inteligencia espacial.- Capacidad de formar imágenes mentales del mundo y manipularlas, implica pensar en tres dimensiones. Capacidad de percibir formas y objetos para resolver problemas que requieren manipulación de imágenes mentales. Gustan de haces mapas conceptuales y mentales. Entienden fácilmente planos y croquis. Es la inteligencia de los navegantes, pilotos de aviación, cirujanos. Las personas que destacan en esta inteligencia gustan dibujar en perspectiva, arman maquetas, manejan espacios, se proyectan en el espacio físico, hacen lectura adecuada de mapas, etc. Hábitos y conductas vinculadas con este tipo de inteligencia: Saber ubicarse y orientarse fácilmente, dibujar y desarrollar buenas perspectivas, gustar de armar rompecabezas o figuras tridimensionales, fotografía, dibujo y pintura, facilidad para explicar algo haciendo bocetos.

Inteligencia cinestésica corporal.- Llamada también físico-kinestésica. Es la inteligencia para solucionar problemas o crear productos utilizando todo el cuerpo o parte de él, como las manos, boca, etc. Implica también la manipulación de objetos y la imitación. Incluye habilidades de coordinación, destreza, equilibrio, flexibilidad, fuerza y velocidad, así como la percepción de medidas y volúmenes. Es la inteligencia de los atletas, bailarines, actores, artesanos, cirujanos, etc. Hábitos y conductas vinculados con este tipo de inteligencia: Andar moviéndose y desplazándose, gustar del deporte, baile y danza, gustar de todo tipo de actividades físicas, expresarse con muchos ademanes, tener buena plasticidad corporal.

Inteligencia interpersonal.- Inteligencia que implica comprender a las personas, interactuar adecuadamente con ellas, motivarlas, comprender sus personalidades, etc. Habilidad para entender los estados de ánimo, motivaciones e intenciones de los demás y de colaborar con ellos. Capacidad para ser sensible a la disposición de los demás. Incluye la sensibilidad a expresiones faciales, la voz, gestos, posturas y habilidad para responder. Esta habilidad es importante para los que trabajan en negocios, enseñanza, medicina, psicología, política, religión. Hábitos y conductas vinculados con este tipo de inteligencia: Gusto pór estar en grupo y participar, asistir a reuniones y fiestas, ser comunicativos con el grupo, conversadores, pueden llegar a ser líderes del grupo, les gusta escuchar y ayudar a otras personas.

Inteligencia intrapersonal.- Capacidad de entenderse a sí mismo con sus fortalezas y debilidades, deseos y miedos. Es un acceso a la propia vida emocional, habilidad para el acceso a la propia vida sentimental, para controlarse y conducirse personalmente, capacidad para mirarse hacia adentro, utilizar la introspección. Incluye la autodisciplina, autocomprensión y autoestima. Se encuentra desarrollada en teólogos, filósofos, psicólogos, personas que son reflexivas, etc. Hábitos y conductas vinculadas con este tipo 
de inteligencia: Gustar de trabajar solos antes que en compañía, ser poco comunicativos con los demás, gustar de la lectura y espacios con poca gente, jugar con escasos amigos, disfrutar de estar y jugar solos. Luego Gardner (2005) agregó la:

Inteligencia naturalista.- Capacidad de distinguir aspectos de la naturaleza, como las diferencias entre una planta y otra, entre animales, nubes, montañas, etc. Incluye habilidades de observación, experimentación, reflexión y cuestionamiento de nuestro entorno. Implica gusto e interés por trabajar en el medio natural. Es la inteligencia de los biólogos, naturistas, ecologistas, agrónomos, etc. Hábitos y conductas vinculadas con este tipo de inteligencia: Gusto por los paseos al campo o a la playa, coleccionar plantas, interesarse en la vida del mundo animal y en general el gusto por investigar características del mudo natural (Del Pozo, 2008). Y después Gardner (2005) sumó la:

Inteligencia existencial.- Inteligencia de los temas trascendentales, de las grandes preguntas, como por ejemplo cuando se medita sobre la muerte, el amor, el conflicto, el futuro del planeta y otras cuestiones existenciales. Gardner afirma que ha especulado sobre esta inteligencia, puesto que está en duda para declararla como una inteligencia totalmente estructurada, puesto que su incertidumbre radica en saber si ciertas regiones del cerebro están dedicadas a la contemplación de estas cuestiones muy generales (Del Pozo, 2008). También se ha mencionado a esta inteligencia como "inteligencia espiritual" considerando las dimensiones de conciencia, trascendencia, gracia, verdad, significado, como por ejemplo en la investigación para la elaboración de un instrumento para su medición presentada por Oliveira, Esgalhado y Pereira (2016).

En el proceso de enseñanza aprendizaje, según Ugarriza (1995), intervienen una serie de factores personales del maestro, del alumno y del contexto social, así como factores del proceso y cambios conductuales. Dentro de los factores de los alumnos están los antecedentes familiares y personales que determinan las características psicológicas y a su vez, estas inciden en el aula como factores del proceso, tanto en los comportamientos del profesor como en los comportamientos de los alumnos. A nivel de cambios conductuales, se observan por ejemplo logros inmediatos en los alumnos con incrementos en conocimientos y habilidades, autoimagen, cambios de actitudes, intereses, capacidades profesionales, personalidad del adulto, etc.

El rendimiento académico puede medirse a través de las notas obtenidas y su promedio histórico a lo largo de los estudios de una carrera profesional. Aunque la evaluación es un proceso complejo. Según Ugarriza (1998) la evaluación es un proceso sistemático dirigido a obtener información exacta y relevante sobre las características de un estudiante, grupo de estudiantes, profesores, materiales, programas, objetivos educativos, etc. Esta información sirve de base para tomar decisiones educacionales, sean de promoción de cursos o grado, de selección y diagnóstico, de innovación o adaptación curricular, de adopción de nuevas técnicas didácticas, de orientación y consejo, o de investigación.

En la presente investigación interesó conocer las inteligencias múltiples en estudiantes de la carrera de administración y gerencia y su posible relación con el rendimiento académico. Se plantearon las siguientes preguntas:

¿Qué características de las inteligencias múltiples presentan los estudiantes de administración y gerencia de una universidad privada de Lima? 
¿Existe relación entre los tipos especificos de inteligencias múltiples y el rendimiento académico de estos estudiantes?

Objetivo general.- Determinar la relación entre las inteligencias múltiples y el rendimiento académico de los estudiantes de administración y gerencia de una universidad privada de Lima.

Objetivos específicos:

Relacionar la inteligencia intrapersonal y el rendimiento académico

Relacionar la inteligencia interpersonal y el rendimiento académico

Relacionar la inteligencia verbal lingüística y el rendimiento académico

Relacionar la inteligencia visual espacial y el rendimiento académico

Relacionar la inteligencia lógico matemática y el rendimiento académico

Relacionar la inteligencia musical rítmica y el rendimiento académico

Relacionar la inteligencia corporal kinestésica y el rendimiento académico

García (2009) comparó las inteligencias múltiples en estudiantes de Publicidad, Relaciones Públicas, Periodismo y Comunicación Audiovisual de Ciencias de la Información de la Universidad Complutense de Madrid, encontrando que todos presentaban altas puntuaciones en todas las inteligencias múltiples, siendo la lingüística la de mayor puntuación y la naturalista la de menor valoración. En segundo lugar, estuvo la interpersonal, en tercero la intrapersonal, en cuarto la espacial. En Publicidad, Relaciones Públicas, Comunicación Audiovisual y en Periodismo la musical. Las mujeres destacaron en interpersonal, mientras que los varones superaron a las mujeres en lingüistica.

Walter, Schneider, Frega y Carvalho de Souza (2009) investigaron las inteligencias múltiples de estudiantes de ciencias contables de la Universidad Estatal del Oeste UNIOESTE de Paraná, relacionándolas con los cursos de administración, geografía, historia y letras y comprando por género. Ciencias contables difería de letras en lógico matemática. Encontraron diferencias en la inteligencia lingüística de historia y letras con ciencias contables y diferencias con geografía en la inteligencia naturalista. Existían diferencias en el desarrollo de la inteligencia lingüística entre segundo y cuarto año, diferencias en inteligencia interpersonal entre primer y segundo año y en segundo y tercer año. No encontraron diferencias en inteligencias múltiples según género.

Rodríguez, Sánchez, Roldán y Franco (2010) investigaron las percepciones de Autoeficacia en Inteligencias Múltiples de 228 ingresantes en el 2009 y 2010 de Ciencias de la Salud de la Universidad Centroccidental Lisandro Alvarado (UCLA), de Medicina (154) y Enfermería (74). Utilizaron el Inventario de Autoeficacia para Inteligencias Múltiples (IAMI) de Fogliatto \& Pérez, encontrándose que los tipos de inteligencia que ocuparon el primer y segundo lugar fueron la intrapersonal e interpersonal, el tercer y cuarto lugar son la lógico matemática y verbal lingüistica, en quinto y sexto se ubicaron la inteligencia naturalista y kinestésica corporal. En los dos últimos lugares se ubicaron la inteligencia visual espacial y musical rítmica, manteniéndose este mismo orden independientemente de la muestra analizada, el programa a que se pertenece, el sexo y la institución de educación de procedencia.

Cerdá, Ortega, Pérez, Flores y Melipillán (2011) estudiaron la relación entre la inteligencia lógica y el rendimiento académico en matemáticas en 4,446 estudiantes de 
educación básica y secundaria de Chile, utilizando el Test de Inteligencia Lógica Superior (TILS) encontrando una correlación positiva y significativa entre la inteligencia lógica con el desempeño académico general y especialmente con el rendimiento académico en la asignatura de matemáticas. En 493 estudiantes talentosos se encontró niveles significativamente mayores de inteligencia lógica.

Gonzáles, Gonzáles, Lauretti y Sandoval (2013) analizaron la inteligencia interpersonal e intrapersonal según el género y la ubicación geográfica, en Caracas (Venezuela) y La Puerta (zona rural y agrícola). Utilizaron el cuestionario de Estimación de la Inteligencia basado en la teoría de las Inteligencias Múltiples de Gardner. Encontraron que las mujeres tuvieron más alta puntuación en inteligencia interpersonal. Las mujeres de La Puerta valoraron inferior en comparación a los hombres en inteligencia intrapersonal. Con referencia a la estimación de sí mismos, los participantes tendieron a valorarse en un nivel más alto que su pareja y que sus hijos. Las hijas mujeres fueron valoradas por sus padres en un nivel inferior con respecto a sus hijos varones.

Stecconi y Kertész (2014) presentaron el Cuestionario de Autoevaluación de las Inteligencias Múltiples (CAIM) aplicado a 813 alumnos de la Universidad de Flores UFLO, de Argentina, de 16 a 60 años, varones y mujeres ingresantes a diversas carreras. Validaron el instrumento y encontraron que los puntajes más altos correspondían a la inteligencia intrapersonal, seguida de las inteligencias interpersonales, verbal, lingüística, corporal kinestésica, lógico matemática, visual espacial y en último lugar la inteligencia musical rítmica.

Salcedo (2015) investigaron a 800 universitarios de la Facultad de Ingeniería de la Universidad Continental de Huancayo, Perú, aplicando el Test de Inteligencias Múltiples de Gardner encontrando mayores inteligencias múltiples por carreras: En Ingeniería Ambiental, de Minas, de Sistemas e Industrial, la inteligencia interpersonal. En Ingeniería Civil, la verbal; en Ingeniería Eléctrica la kinestésica. Encontró correlación significativa entre inteligencia interpersonal y rendimiento académico en la carrera de Ingeniería Ambiental y correlación significativa entre inteligencia verbal y rendimiento académico de Ingeniería Civil.

Barraza y Gonzáles (2016) investigando a 252 estudiantes de la Universidad Santo Tomás- La Serena, de Chile, aplicado la escala MIDAS-teens de Shearer traducida y adaptada para Chile por Pizarro, Redondo, Castillo, Alarcón y Saavedra, encontraron correlaciones débiles entre el rendimiento académico y la autopercepción de las inteligencias múltiples: Lingüística y lógico matemática.

Los tipos de inteligencia tienen implicancias para el proceso de enseñanza aprendizaje. El Programa Nacional Aprender Enseñando (2016) y Fonseca Mora (2002) presentan cómo los alumnos con marcada tendencia específica de inteligencia múltiple piensan y necesitan las siguientes herramientas didácticas, recursos y materiales que pueden utilizarse en la enseñanza para potenciar las inteligencias múltiples (Vargas, 2004; Suárez, Maíz y Meza, 2010): 
Tabla 1

Cómo piensan y qué necesitan los alumnos con marcada tendencia de inteligencia múltiple. Adaptado de Fonseca Mora (2002), Vargas (2004), Suárez, Maíz y Meza (2010), Programa Nacional Aprender Enseñando (2016).

\begin{tabular}{ccc}
\hline $\begin{array}{c}\text { Inteligencia } \\
\text { múltiple }\end{array}$ & Piensan & Necesitan \\
\hline
\end{tabular}

\begin{tabular}{|c|c|c|}
\hline $\begin{array}{l}\text { Verbal } \\
\text { Lingüística }\end{array}$ & En palabras & $\begin{array}{l}\text { Libros, elementos para escribir, papel, confecciones de } \\
\text { diarios, memorización de hechos lingüísticos, diálogo, } \\
\text { discusión en grupos grandes y pequeños, debates, } \\
\text { cuentos, narraciones, exposiciones orales, hojas de } \\
\text { trabajo, reuniones creativas, juegos de palabras, } \\
\text { publicaciones, elaboración de un periódico mural. } \\
\text { Cosas para explorar y pensar, materiales de ciencias, }\end{array}$ \\
\hline $\begin{array}{l}\text { Lógico } \\
\text { Matemática }\end{array}$ & $\begin{array}{l}\text { Por medio del } \\
\text { razonamiento }\end{array}$ & $\begin{array}{l}\text { cosas para manipular, visitas al planetario, visitas } \\
\text { al museo de ciencias, demostraciones científicas, } \\
\text { ejercicios para resolver problemas lógicos, clasificación } \\
\text { y agrupaciones, juegos y rompecabezas de lógica, } \\
\text { cálculos mentales, pensamiento crítico. }\end{array}$ \\
\hline Visual Espacial & $\begin{array}{l}\text { En imágenes y } \\
\text { fotografías }\end{array}$ & $\begin{array}{l}\text { Arte, cuadros, gráficos, diagramas, mapas, mapas } \\
\text { mentales, metáforas, fotografías, "ladrillos de juguete", } \\
\text { videos, películas, diapositivas, juegos de imaginación, } \\
\text { laberintos visuales, modelos tridimensionales, ejercicios } \\
\text { de pensamiento visual, rompecabezas, libros ilustrados, } \\
\text { visitas a museos. }\end{array}$ \\
\hline $\begin{array}{l}\text { Corporal } \\
\text { Kinestésica }\end{array}$ & $\begin{array}{l}\text { Por medio de } \\
\text { sensaciones } \\
\text { somáticas }\end{array}$ & $\begin{array}{l}\text { Juegos de actuación, teatro, dramatizaciones, } \\
\text { danzas, movimientos, cosas para construir, deportes, } \\
\text { juegos físicos, experiencias táctiles, experiencias de } \\
\text { aprendizaje directas, excursiones, pantomimia, uso de } \\
\text { imágenes cinestésicas, software de realidad virtual, } \\
\text { ejercicios de relajación. }\end{array}$ \\
\hline Musical Rítmica & $\begin{array}{l}\text { Por medio de } \\
\text { ritmos y melodías }\end{array}$ & $\begin{array}{l}\text { Tiempos dedicados al canto, canciones didácticas, } \\
\text { tarareo, grabaciones, elaboración de diferentes ritmos, } \\
\text { música de fondo, creación de melodías, software para } \\
\text { música, ejercicios de memoria musical, narración } \\
\text { cantada, juegos rítmicos, asistencia a conciertos, tocar } \\
\text { instrumentos musicales. }\end{array}$ \\
\hline Interpersonal & $\begin{array}{l}\text { Intercambiando } \\
\text { ideas con otras } \\
\text { personas }\end{array}$ & $\begin{array}{l}\text { Amigos, grupos cooperativos, mediación de conflictos, } \\
\text { reuniones creativas, juegos grupales, reuniones } \\
\text { sociales, participación en la comunidad, festividades } \\
\text { comunales, fiestas, clubes, aprendizaje tipo maestro/ } \\
\text { aprendiz. }\end{array}$ \\
\hline
\end{tabular}


Intrapersonal Muy íntimamente Juegos individualizados, reflexiones, conexiones personales, actividades de autoestima, confección de diarios, sesiones de definición de metas, lugares secretos, tiempo para estar solo, proyectos manejados a su propio ritmo, alternativas, visualización, relajación.

\section{Hipótesis General}

$H_{1}$ Existen relaciones significativas entre los índices de inteligencias múltiples y el rendimiento académico de estudiantes de administración y gerencia de una universidad privada de Lima.

\section{Hipótesis Específicas}

$H_{1.1}$ Existe relación positiva entre la inteligencia intrapersonal y el rendimiento académico

$\mathrm{H}_{1.2}$ Existe relación positiva entre la inteligencia interpersonal y el rendimiento académico

$H_{1.3}$ Existe relación positiva entre la inteligencia verbal lingüística y el rendimiento académico

$\mathrm{H}_{1.4}$ Existe relación positiva entre la inteligencia visual espacial y el rendimiento académico

$H_{1.5}$ Existe relación positiva entre la inteligencia lógico matemática y el rendimiento académico

$\mathrm{H}_{1.6}$ Existe relación positiva entre la inteligencia musical rítmica y el rendimiento académico

$\mathrm{H}_{1.7}$ Existe relación positiva entre la inteligencia corporal kinestésica y el rendimiento académico

\section{Metodología del Estudio}

Tipo y método de investigación.- Tipo básica (Sánchez y Reyes, 2015) pues se trata de proporcionar conocimientos sobre un área del saber humano. El método es descriptivo. La investigación es correlacional en el sentido de que busca relacionar las 2 variables: Rendimiento académico e inteligencias múltiples.

Población de estudio.- 720 estudiantes de la carrera de administración y gerencia de la Facultad de Ciencias Económicas y Empresariales de una universidad privada de la ciudad de Lima.

Diseño muestral.- 100 varones (48\%) y mujeres (52\%), según la tabla 3, seleccionados de forma intencional entre los universitarios de la carrera de administración y gerencia, del 6 to al 10 mo semestre. En la tabla 2 se observa las edades: el 58\% de la muestra tenía entre 20 a 23 años y $31 \%$ entre 24 a 27 años, siendo la mayor edad la comprendida entre 32 a 35 años, con $2 \%$ de los participantes. 
Tabla 2

Edades de la muestra participante en la investigación

\begin{tabular}{lll}
\hline Edad & $\mathrm{f}$ & $\%$ \\
\hline $20-23$ & 58 & 58 \\
$24-27$ & 31 & 31 \\
$28-31$ & 9 & 9 \\
$32-35$ & 2 & 2 \\
Total & 100 & 100 \\
\hline
\end{tabular}

Tabla 3

Sexo de la muestra participante en la investigación

\begin{tabular}{lll}
\hline Sexo & $\mathrm{f}$ & $\%$ \\
\hline Varones & 48 & 48 \\
Mujeres & 52 & 52 \\
Total & 100 & 100 \\
\hline
\end{tabular}

Técnicas e instrumentos de recolección de datos.- Cuestionario de Autoevaluación de las Inteligencias Múltiples (CAIM) del Dr. Roberto Kestész, de la Universidad de Flores, de Argentina (Kertész, 2014; Stecconi y Kertész, 2014), que evalúa los siguientes tipos de inteligencias múltiples:
Intrapersonal
(lo que ocurre en la mente)
Interpersonal (las relaciones con los demás)
Verbal lingüística (leer, escribir, comunicarse hablando)
Visual espacial (imaginar, observar, crear formas)
Lógico matemática (abstracción, razonamiento, cálculo)
Musical rítmica (oído musical, tonos de voz, sonidos, ritmos)
Corporal kinestésica (percepción y control del propio cuerpo, expresión física, habilidad manual)

La adaptación del instrumento se realizó para estudiantes de Lima. La validez se obtuvo por criterio de expertos, con seis psicólogos jueces, utilizándose el índice de Lawshe (1975), por el cual todos los ítems fueron aceptados.

Para el cálculo de la confiabilidad del instrumento se realizó una prueba piloto con 35 estudiantes de Administración y Gerencia, utilizándose el estadístico Alfa de Cronbach, encontrándose 2 items que fueron eliminados, según se observa en la tabla № 4: El ítem № 2 del área de Inteligencia Interpersonal "Escucho a los demás y llego a aceptarlos aunque no estéde acuerdo con ellos" y el ítem № 4 del área de Inteligencia Verbal Lingüística "Expreso fácilmente hablando lo que pienso y siento, en forma comprensible para otros". 
Tabla 4

Alfa de Cronbach para el análisis de confiabilidad del Cuestionario Inteligencias Múltiples CAIM de Kestész

\begin{tabular}{|c|c|c|c|c|c|}
\hline Items & Media & Varianza & $\begin{array}{l}\text { Correlación } \\
\text { Corregida }\end{array}$ & Item & Test Alfa de Cronbach \\
\hline Item INTRA1 & 72.37 & 103.358 & .254 & & .825 \\
\hline Item INTRA2 & 71.43 & 100.723 & .375 & & .816 \\
\hline Item INTRA3 & 71.97 & 86.617 & 692 & & .783 \\
\hline Item INTRA4 & 71.66 & 80.467 & 646 & & .787 \\
\hline Item INTRA5 & 71.74 & 98.903 & .369 & & .816 \\
\hline Item INTRA6 & 72.00 & 88.941 & 687 & & .786 \\
\hline Item INTRA7 & 71.63 & 101.887 & .215 & & .832 \\
\hline Item INTRA8 & 72.66 & 86.703 & .539 & & .801 \\
\hline Item INTRA9 & 72.54 & 89.726 & .508 & & .804 \\
\hline Item INTRA10 & 72.26 & 84.844 & .744 & & .777 \\
\hline Item INTER1 & 66.37 & 59.182 & .407 & & .774 \\
\hline Item INTER2 & 74.80 & 70.282 & .066 & & $.786 *$ \\
\hline Item INTER3 & 67.63 & 51.770 & .519 & & .760 \\
\hline Item INTER4 & 67.20 & 58.400 & .437 & & .770 \\
\hline Item INTER5 & 67.31 & 53.634 & .493 & & .763 \\
\hline Item INTER6 & 65.49 & 64.845 & .375 & & .780 \\
\hline Item INTER7 & 65.71 & 58.916 & .447 & & .769 \\
\hline Item INTER8 & 66.26 & 54.903 & .551 & & .754 \\
\hline Item INTER9 & 65.80 & 54.929 & .614 & & .746 \\
\hline Item INTER10 & 66.63 & 56.593 & .455 & & .768 \\
\hline Item VL1 & 61.83 & 76.029 & .572 & & .792 \\
\hline Item VL2 & 61.46 & 73.667 & .605 & & .787 \\
\hline Item VL3 & 60.80 & 81.635 & .262 & & .823 \\
\hline Item VL4 & 69.26 & 90.844 & .155 & & $.815 *$ \\
\hline Item VL5 & 62.20 & 74.165 & .423 & & .809 \\
\hline Item VL6 & 61.23 & 70.534 & .633 & & .781 \\
\hline Item VL7 & 61.43 & 67.017 & .790 & & .761 \\
\hline Item VL8 & 62.11 & 73.634 & .452 & & .805 \\
\hline Item VL9 & 61.43 & 71.134 & .421 & & .814 \\
\hline Item VL10 & 61.57 & 73.134 & .569 & & .790 \\
\hline Item VE1 & 64.51 & 220.081 & .783 & & .906 \\
\hline Item VE2 & 63.40 & 262.953 & .442 & & .924 \\
\hline Item VE3 & 62.91 & 254.139 & .664 & & .912 \\
\hline Item VE4 & 63.63 & 232.358 & .861 & & .900 \\
\hline Item VE5 & 62.03 & 256.734 & .716 & & .911 \\
\hline Item VE6 & 63.74 & 247.844 & 699 & & .910 \\
\hline Item VE7 & 63.80 & 253.871 & .630 & & .914 \\
\hline Item VE8 & 63.29 & 238.387 & .719 & & .909 \\
\hline Item VE9 & 63.40 & 238.012 & .754 & & .907 \\
\hline
\end{tabular}




\begin{tabular}{|c|c|c|c|c|}
\hline Item VE10 & 63.14 & 240.538 & .744 & .907 \\
\hline Item LM1 & 61.23 & 177.711 & .321 & .906 \\
\hline Item LM2 & 62.14 & 159.655 & .779 & .878 \\
\hline Item LM3 & 61.57 & 166.664 & .641 & .886 \\
\hline Item LM4 & 63.17 & 153.676 & .741 & .879 \\
\hline Item LM5 & 61.86 & 165.832 & .678 & .884 \\
\hline Item LM6 & 63.11 & 157.398 & .675 & .884 \\
\hline Item LM7 & 62.14 & 161.891 & .708 & .882 \\
\hline Item LM8 & 63.83 & 142.205 & .708 & .885 \\
\hline Item LM9 & 62.17 & 163.852 & .664 & .885 \\
\hline Item LM10 & 61.83 & 167.676 & .607 & .888 \\
\hline Item MR1 & 58.86 & 316.361 & .664 & .899 \\
\hline Item MR2 & 58.49 & 322.787 & .745 & .897 \\
\hline Item MR3 & 57.80 & 350.341 & .434 & .911 \\
\hline Item MR4 & 59.20 & 311.341 & .723 & .896 \\
\hline Item MR5 & 58.20 & 335.400 & .599 & .904 \\
\hline Item MR6 & 60.34 & 282.997 & .774 & .893 \\
\hline Item MR7 & 61.77 & 288.240 & .706 & .898 \\
\hline Item MR8 & 61.54 & 286.255 & .779 & .892 \\
\hline Item MR9 & 60.71 & 284.975 & .856 & .886 \\
\hline Item MR10 & 58.89 & 332.045 & .482 & .909 \\
\hline Item CK1 & 62.91 & 174.081 & .291 & .856 \\
\hline Item CK2 & 64.17 & 160.205 & .352 & .859 \\
\hline Item CK3 & 63.63 & 168.476 & .387 & .851 \\
\hline Item CK4 & 63.60 & 148.424 & .780 & .819 \\
\hline Item CK5 & 63.91 & 152.257 & .634 & .831 \\
\hline Item CK6 & 63.63 & 144.358 & .677 & .826 \\
\hline Item CK7 & 64.83 & 146.323 & .626 & .831 \\
\hline Item CK8 & 64.49 & 152.963 & .756 & .824 \\
\hline Item CK9 & 64.34 & 150.114 & .588 & .835 \\
\hline Item CK10 & 64.00 & 154.529 & .521 & .841 \\
\hline
\end{tabular}

*Item suprimido por falta de confiabilidad

Luego de la eliminación de los 2 items, el área de inteligencia interpersonal y el área de inteligencia verbal lingüística, quedaron cada una con 9 items para evaluación y con un cálculo del Alfa de Cronbach estadísticamente significativos según se aprecia en la tabla 5. 
Tabla 5

Alfa de Cronbach para el análisis de confiabilidad del Cuestionario de Inteligencias Múltiples CAIM de R. Kertész

\begin{tabular}{lll}
\hline Tipo de Inteligencia & No Items & Alfa de Cronbach \\
\hline Intrapersonal & 10 & .820 \\
Interpersonal & 9 & .786 \\
Verbal Lingüística & 9 & .815 \\
Visual Espacial & 10 & .919 \\
Lógico Matemática & 10 & .896 \\
Musical Rítmica & 10 & .908 \\
Corporal Kinestésica & 10 & .852 \\
$\mathrm{~N}$ & & 35 \\
\hline
\end{tabular}

Procedimiento para la recolección de datos.- El Cuestionario de Autoevaluación de las Inteligencias Múltiples (CAIM) se aplicó en un estudio piloto a 35 estudiantes de la carrera de administración y gerencia, del 6to al $10 \mathrm{mo}$ semestre de estudios, de forma colectiva en sus aulas de clases. Como producto de ese estudio se realizaron los análisis estadísticos de confiabilidad del instrumento, por el cual se eliminaron 2 items del cuestionario. Con el instrumento reformulado se aplicó el cuestionario a la muestra definitiva en los meses de octubre y noviembre 2016. En todos los casos previamente la muestra firmó un "Consentimiento informado" para participar en la investigación. Las notas de rendimiento académico fueron proporcionadas por la Oficina de Registros y Matrícula de la Facultad, considerándose el promedio histórico del alumno, en un sistema de evaluación vigesimal de 0 a 20.

Técnicas de procedimiento y análisis de datos.- Los datos se procesaron con el programa estadístico del SPSS, considerando los puntajes obtenidos en las variables investigadas. El estadístico elegido fue el coeficiente de correlación de Pearson, con un nivel de significatividad del $0.05 \%$ de probabilidad.

\section{Resultados}

En el análisis de los datos descriptivos de las variables de la investigación se puede observa en la tabla 6 que la nota promedio de los estudiantes es 12.1894. Con respecto a las inteligencias múltiples se encuentra un mayor puntaje en la Intrapersonal (82.09) y un menor puntaje en la Musical Rítmica (68.4). 
Natividad Espíritu Salinas; Jorge Carbajal Arroyo; William Torres Acuña; José Canales Sierralta

Y AMÉRICO SOTO GRIMALDO

Tabla 6

Estadística descriptiva de las variables estudiadas, en alumnos de administración y gerencia

\begin{tabular}{llll}
\hline \multicolumn{1}{c}{ Variable } & \multicolumn{1}{c}{ Media } & \multicolumn{1}{c}{$\begin{array}{c}\text { Desviación } \\
\text { estándar }\end{array}$} & $\mathrm{N}$ \\
\hline Rendimiento académico (promedio notas histórico) & 12.1894 & 1.50130 & 100 \\
Inteligencia Intrapersonal & 82.09 & 9.521 & 100 \\
Inteligencia Interpersonal & 74.86 & 7.661 & 100 \\
Inteligencia Verbal Lingüística & 68.73 & 11.481 & 100 \\
Inteligencia Visual Espacial & 70.74 & 15.912 & 100 \\
Inteligencia Lógico Matemática & 72.10 & 13.478 & 100 \\
Inteligencia Musical Rítmica & 68.40 & 19.077 & 100 \\
Inteligencia Corporal Kinestésica & 73.23 & 15.716 & 100 \\
\hline
\end{tabular}

Con respecto a la Hipótesis General, se encontró que no existen relaciones significativas entre los índices de inteligencia múltiple y el rendimiento académico de los estudiantes, según se puede observar en las siguientes y sucesivas tablas del cálculo de la correlación de Pearson. La hipótesis específica 1 no fue aceptada al obtenerse una correlación de 0.038 , siendo inferior a 0.705 , que es el puntaje de probabilidad al $0.05 \%$ de significatividad, es decir, el rendimiento académico no se relaciona con la Inteligencia Intrapersonal, según se observa en la tabla № 7 . Igualmente no se ha encontrado relación significativa entre el rendimiento académico y la Inteligencia Interpersonal (Tabla 8), por lo que no se acepta la hipótesis específica 2. Con respecto a la Hipótesis Específica 3, no se acepta, porque se encuentra que no hay relación entre el rendimiento académico y la Inteligencia Verbal Lingüística, al resultar una correlación de Pearson de 0.061, según la tabla № 9.

Tabla 7

Cálculo de la relación entre Rendimiento Académico y el componente de la Inteligencia Intrapersonal del CAIM utilizando la prueba de Pearson.

\begin{tabular}{llll}
\hline & Inteligencia Intrapersonal CAIM & $\mathrm{N}$ & $\mathrm{p}$ \\
\hline Rendimiento Académico & 0.038 & 100 & 0.705 \\
\hline${ }^{*} \mathrm{p}<0.05$ & &
\end{tabular}

Tabla 8

Cálculo de la relación entre Rendimiento Académico y el componente de la Inteligencia Interpersonal del CAIM utilizando la prueba de Pearson.

\begin{tabular}{llll}
\hline & Inteligencia Interpersonal CAIM & $\mathrm{N}$ & $\mathrm{p}$ \\
\hline Rendimiento Académico & 0.017 & 100 & 0.868 \\
\hline${ }^{*} \mathrm{p}<0.05$ & & &
\end{tabular}


Tabla 9

Cálculo de la relación entre Rendimiento Académico y el componente de la Inteligencia Verbal Lingüística del CAIM utilizando la prueba de Pearson.

\begin{tabular}{llll}
\hline & Inteligencia Verbal Lingüística CAIM & $\mathrm{N}$ & $\mathrm{p}$ \\
\hline Rendimiento Académico & 0.061 & 100 & 0.548 \\
\hline
\end{tabular}

${ }^{*} p<0.05$

No se acepta la hipótesis específica 4, pues no es significativa la relación entre el rendimiento académico y la inteligencia visual espacial (tabla 10). La hipótesis específica 5 no es aceptada puesto que no hay relación entre el rendimiento académico y la inteligencia lógico matemática (tabla 11).

Tabla 10

Cálculo de la relación entre Rendimiento Académico y el componente de la Inteligencia Visual Espacial del CAIM utilizando la prueba de Pearson.

\begin{tabular}{llll}
\hline & Inteligencia Visual Espacial CAIM & $\mathrm{N}$ & $\mathrm{p}$ \\
\hline Rendimiento Académico & 0.046 & 100 & 0.65 \\
\hline
\end{tabular}

* $p<0.05$

Tabla 11

Cálculo de la relación entre Rendimiento Académico y el componente de la Inteligencia Lógico Matemática del CAIM utilizando la prueba de Pearson.

\begin{tabular}{llll}
\hline & Inteligencia Lógico Matemática CAIM & $\mathrm{N}$ & $\mathrm{p}$ \\
\hline Rendimiento Académico & 0.132 & 100 & 0.191 \\
\hline
\end{tabular}

${ }^{*} p<0.05$

La hipótesis específica 6 no es aceptada porque no existe relación significativa entre el rendimiento académico y la inteligencia musical rítmica (tabla 12). Finalmente la hipótesis específica 7 no fue aceptada porque no existe correlación significativa entre el rendimiento académico y la inteligencia corporal kinestésica (tabla 13).

Tabla 12

Cálculo de la relación entre Rendimiento Académico y el componente de la Inteligencia Musical Rítmica del CAIM utilizando la prueba de Pearson.

\begin{tabular}{llll}
\hline & Inteligencia Musical Rítmica CAIM & $\mathrm{N}$ & $\mathrm{p}$ \\
\hline Rendimiento Académico & -0.066 & 100 & 0.515 \\
\hline${ }^{*} \mathrm{p}<0.05$ & & &
\end{tabular}


Tabla 13

Cálculo de la relación entre Rendimiento Académico y el componente de la Inteligencia Corporal Kinestésica del CAIM utilizando la prueba de Pearson.

\begin{tabular}{llll}
\hline & Inteligencia Corporal Kinestésica CAIM & $N$ & $p$ \\
\hline Rendimiento Académico & -0.122 & 100 & 0.225 \\
\hline${ }^{*} \mathrm{p}<0.05$ & & &
\end{tabular}

\section{Discusión}

Las inteligencias múltiples no tienen relación estadística significativa específica con el rendimiento académico en una muestra homogénea de estudiantes de administración y gerencia de una universidad privada de Lima. Esta conclusión, a la que se arriba con la presente investigación, no es generalizable a otras muestras, pues corresponde a un análisis muy fino que se ha realizado en participantes que, se supone, todos ellos tienen una inteligencia general normal o superior al promedio, puesto que el examen de admisión para la selección al ingreso a la universidad permite filtrar a los postulantes y aceptar para los estudios superiores sólo a aquellos que tienen condiciones intelectuales para seguir una carrera a nivel superior. Además se supone que la mayoría de ellos tenían intereses vocacionales homogéneos que los impulsó a estudiar la carrera elegida de administración y gerencia. Barraza y Gonzáles (2016) también habían encontrado correlaciones débiles entre el rendimiento académico y la autopercepción de las inteligencias múltiples: Lingüística y lógico matemática.

Sin embargo, a pesar de no ser estadísticamente significativa, se ha encontrado relación positiva entre el rendimiento académico y las inteligencias múltiples Intrapersonal, Interpersonal, Verbal Lingüística, Visual Espacial y Lógico Matemática. La correlación fue negativa, aunque estadísticamente no significativa, entre el rendimiento académico y las inteligencias Musical Rítmica y Corporal Kinestésica. Es probable que la relación negativa encontrada se deba al tipo de carrera de estudio: Administración y gerencia. Los hallazgos hubieran sido distintos por ejemplo si se tratara de estudiantes de música, danzas, deportes, etc.

Si se hubiera comparado las inteligencias múltiples con estudiantes de otras carreras posiblemente se encontrarían diferencias estadísticamente significativas. Sin embargo esto no ha sido el objetivo de la presente investigación. Por ejemplo ¿Se hubieran encontrado diferencias de las inteligencias múltiples entre los estudiantes de administración y gerencia, comparando con ingeniería, psicología, ciencias biológicas, arquitectura, contabilidad, medicina, etc.?

También es necesario considerar que del instrumento original CAIM, argentino, del Dr. Roberto Kestész, en un análisis de confiabilidad para la muestra peruana, se eliminaron el ítem №2 del área de Inteligencia Interpersonal "Escucho a los demás y llego a aceptarlos aunque no esté de acuerdo con ellos" y el ítem 4 del área de Inteligencia Verbal-Lingüística "Expreso fácilmente hablando lo que pienso y siento, en forma comprensible para otros", porque tal vez no fueron entendidos en toda su magnitud por la muestra peruana, por factores de 
diferencias culturales. Pero, por otro lado, en la presente investigación se han encontrado los mismos resultados que la investigación de Stecconi y Kertèsz (2014), quienes al aplicar el cuestionario CAIM en 813 alumnos ingresantes a la Universidad de Flores, de Argentina, encontraron los puntajes más altos en el tipo de inteligencia Intrapersonal y los más bajos en la inteligencia Musical-Rítmica.

En varias investigaciones anteriores se ha encontrado que el factor de inteligencia no es el determinante para el rendimiento académico. Con respecto a la inteligencia general, en la investigación de Canales (2005) los estudiantes de psicología de alto y bajo rendimiento académico de una universidad privada de Lima no tenían diferencias significativas intelectuales, medidas con el Test de Matrices Progresivas de Raven, pero sí tenían diferencias estadísticamente significativas en la cantidad de conductas parentales negativas ("frenadores" en la teoría del Análisis Transaccional) que habían recibido en su infancia. Los alumnos de bajo rendimiento académico reportaron significativamente más conductas parentales negativas en la infancia. Se podría argumentar que existen otras variables, como las emocionales, que influyen más en el rendimiento académico que la misma inteligencia.

Por otro lado, los hábitos de estudio parecen ser determinantes para el rendimiento académico, según lo demuestra por ejemplo el trabajo de Escurra, Romero, Moreno, Ahumada, Juarez y Ramos (2016), quienes al investigar a estudiantes de estadística e informática de una universidad peruana encontraron relación moderada entre los hábitos y actitudes generales de trabajo, así como hábitos de estudio, con el rendimiento académico. Trejo, Castañeda, Valverde y Cruz (2016) encontraron que la didáctica universitaria, la metodología del docente y los recursos didácticos que utiliza el docente se relacionan significativamente con el rendimiento académico de los estudiantes de la carrera de bromatología y nutrición de la Universidad José Faustino Sánchez Carrión, de Perú.

Pesantes, Valencia, Falcon, Albitres y Manes (2016) determinaron que existe relación entre la actitud hacia la matemática y el rendimiento académico de los estudiantes del I ciclo de la facultad de Bromatología y Nutrición de la Universidad Nacional José Faustino Sánchez Carrión. Campo, Escorcia, Moreno y Palacio (2016) encontraron una correlación positiva entre el nivel de rendimiento y los conocimientos metacognitivos en estudiantes universitarios colombianos y franceses de psicología. La metacognición involucra operaciones o estrategias de autorregulación como la planeación de la tarea, la supervisión de los procesos cognitivos que se activan durante la realización de dicha tarea y la evaluación de los resultados.

En el proceso de enseñanza aprendizaje, según Ugarriza (1995), intervienen una serie de factores personales del maestro, del alumno y del contexto social, así como factores del proceso y cambios conductuales. Los factores de los alumnos, dentro de lo cual están los antecedentes familiares y personales que determinan las características psicológicas y a su vez, estas inciden en el aula como factores del proceso, tanto en los comportamientos del profesor como en los comportamientos de los alumnos. A nivel de cambios conductuales, se observan por ejemplo logros inmediatos en los alumnos con incrementos en conocimientos y habilidades, autoimagen, cambios de actitudes, intereses, capacidades profesionales, personalidad del adulto, etc. 
Más que el factor de inteligencia general o inteligencias múltiples, la educación debe incidir en los aspectos personales, en la salud mental, en la estabilidad emocional del estudiante, en sus hábitos de estudio, en los aspectos metacognitivos, la didáctica docente, las actitudes de los estudiantes hacia materias de estudio específicas, etc., aspectos que serían motivo de estudio de otras investigaciones.

\section{Conclusiones}

1. El rendimiento académico de la muestra estudiada de administración y gerencia, representado por el promedio histórico de notas, es de 12.19 en un sistema de evaluación vigesimal.

2. La inteligencia múltiple más desarrollada es la Inteligencia Intrapersonal (82.09)

3. La inteligencia múltiple menos desarrollada es la Inteligencia Musical Rítmica (68.4)

4. Existe correlación positiva entre el rendimiento académico y las inteligencias múltiples Intrapersonal, Interpersonal, Verbal Lingüística, Visual Espacial y Lógico Matemática, sin embargo esta relación no es estadísticamente significativa.

5. Existe correlación negativa entre el rendimiento académico y las inteligencias Musical Rítmica y Corporal Kinestésica, pero es estadísticamente no significativa.

\section{Referencias}

Barraza, R. y Gonzáles, M. (2016). Rendimiento académico y autopercepción de inteligencias múltiples e inteligencia emocional en universitarios de primera generación. Revista Electrónica Actualidades Investigativas en Educación, 19(2),1-23. Universidad de Costa Rica. San Pedro de Montes de Oca, Costa Rica. Recuperado: http://revista.inie.ucr.ac.cr/ index.php/aie/article/viewFile/1274/1142

Canales, J.A. (2005). Estudio comparativo de las Conductas Parentales Negativas entre estudiantes universitarios de Psicología de alto y bajo rendimiento académico a través del Análisis Transaccional. (Tesis inédita para optar el Grado de Maestro en Docencia Superior), Universidad Ricardo Palma, Lima, Perú.

Carpintero, E., Cabezas, D. y Pérez, L. (2009). Inteligencias múltiples y altas capacidades. Una propuesta de enriquecimiento basada en el modelo de Howard Gardner. Faísca, 14(16), 4-13.

Cerda, G., Ortega, R., Pérez, C., Flores, C. y Melipillán, R. (2011). Inteligencia lógica y rendimiento académico en matemáticas: un estudio con estudiantes de Educación Básica y Secundaria de Chile. Anales de Psicología, 27(2), 389-398 Universidad de Murcia, España. Recuperado de: http://www.redalyc.org/pdf/167/16720051015.pdf

Cossio, A. (2007). Apoyemos a desarrollar el espíritu creativo de los niños mediante sus inteligencias múltiples. Maestros y alumnos. Revista de la Escuela de Educación. Facultad de Psicología Universidad Ricardo Palma, Lima. 3(3).

Del Pozo, M. (2008). Inteligencias Múltiples. Howard Gardner en el Colegio Montserrat. Cuadernos de Pedagogía №376. Febrero. № identificador: 376.012 
Ferrandiz, C., Prieto, M., Bermejo, M. y Ferrando, M. (2006). Fundamentos psicopedagógicos de las inteligencias múltiples. Revista española de pedagogía. Año LXIV №233 eneroabril, 5-20

Fonseca Mora, M. C. (2002). Inteligencias Múltiples, Múltiples Formas de enseñar Inglés. Sevilla: Mergablum.

García, M. (2009) La dimensión comunicativa de las inteligencias múltiples. CIC Cuadernos de Información y comunicación, 14, 141-157

Gardner, H. (1983). Estructuras de la mente: la teoría de las Inteligencias Múltiples. Colombia: Fondo de Cultura Económica.

Gardner, H. (1988). Inteligencias Múltiples, La teoría con la práctica. (Octava Edición). España: Editorial Paidós Ibérica.

Gardner, H. (1993). Inteligencias Múltiples, New York: Editorial Basic Books.

Gardner, H. y Hatch, T. (1989). Multiple Intelligences Go To School: Educational Implications of the Theory of Multiple Intelligences. Educational Researcher, 18(8), 4-9.

Gardner, H. (2005). Múltiples lentes sobre la mente. Separata. Sinéctica. Trabajo presentado en la Conferencia de Expo Gestión, Bogotá, Colombia. Recuperado de: http://ezproxy. concytec.gob.pe:2096/ehost/pdfviewer/pdfviewer?vid=5\&sid=35200fc3-7e7b-4bfd9b12-5fcab6eff3f5\%40sessionmgr4006\&hid=4206

Gardner, H. (2013). Harvard Project Zero: A Personal History. Recuperado de: https:// howardgardner01.files.wordpress.com/2012/06/pz-history-9-10-13.pdf

Gonzáles, L., Gonzáles, O., Lauretti, P. y Sandoval, A. (2013). Estimación de la inteligencia interpersonal e intrapersonal según el género y la ubicación geográfica. Psicogente, 16(30): 368-378. Julio Diciembre. Universidad Simón Bolívar. Barranquilla, Colombia

Gonzales, L. y López, G. (2006). Inteligencia y Educación. Revista Panamericana de Pedagogía. No $8,155-211$

Kertész, R. (2014). Cuestionario de Autoevaluación de las inteligencias múltiples (C.A.I.M.) Buenos Aires: Instituto Privado de Psicología Médica S.A. IPPEM.

Lawshe, C. H. (1975). A quantitative approach to content validity. Personnel Psychology, 28, 563-575

Macías, M. (2002). Las múltiples inteligencias. Psicología desde el Caribe. (10), 27-38. Universidad del Norte, Barranquilla, Colombia. Recuperado de: http://www.redalyc. org/pdf/213/21301003.pdf

Oliveira, D., Esgalhado, G. y Pereira, H. (2016). Inteligência espiritual: Validação preliminar da versão portuguesa da Escala de Inteligência Espiritual Integrada (EIEI). Análise Psicológica, 3 (XXXIV), 325-337 doi: 10.14417/ap.982

Programa Nacional Aprender Enseñando (2016). Inteligencias Múltiples. Consultado en: http://www.psicologia.unt.edu.ar/wp-content/uploads/2016/04/I-M-Actividades.pdf

Rodríguez, A., Sánchez, G., Roldán, O. y Franco, M. (2010). Percepción de autoeficacia en inteligencias múltiples de los estudiantes de nuevo ingreso al Decanato de Ciencias de la Salud. UCLA. Académica. 2(3), 141-154.

Salcedo, J. (2016) Inteligencias múltiples y rendimiento académico de estudiantes universitarios en Huancayo, 2015. Apuntes de Ciencias Sociales, 06 (01). doi: http:// dx.doi.org/10.18259/acs.2016005. 
Sánchez, H. (2007).Cómo desarrollarel pensamiento creativo. Una propuestaPsicopedagógica. Lima: Editorial Visión Universitaria.

Sánchez, H. y Reyes, C. (2015). Metodología y Diseños en la Investigación Científica. Lima: Business Support Aneth.

Stecconi, C. y Kertész, R. (2014). El cuestionario de autoevaluación de las inteligencias múltiples: su utilización en el desarrollo del potencial personal. VI Congreso Internacional de Investigación y Práctica Profesional en Psicología XXI Jornadas de Investigación Décimo Encuentro de Investigadores en Psicología del MERCOSUR. Facultad de Psicología - Universidad de Buenos Aires, Buenos Aires, 205-208.

Sternberg, R. (2015). Successful intelligence: A model for testing intelligence beyond IQ tests. European Journal of Education and Psychology, 8, 76-84. Recuperado de: http:// www.sciencedirect.com/science/article/pii/S1888899215000070

Suárez, J., Maíz, F. y Meza, M. (2010). Inteligencias múltiples: una innovación pedagógica para potenciar el proceso enseñanza aprendizaje. Investigación y Postgrado, 25(1), enero-junio 81-94 Universidad Pedagógica Experimental Libertador. Recuperado de: http://www.redalyc.org/articulo.oa?id=65822264005

Ugarriza, N. (1995). Rendimiento académico del alumno de la URP según modalidad de admisión y traslado interno promoción 1988-I, 1992-II, Informe Final. Oficina de Registros y Coordinación Académica de la Universidad Ricardo Palma. Lima, Perú.

Ugarriza, N. (1998). Evaluación del rendimiento académico. Revista Educación Superior de la Unidad de Post Grado de la Facultad de Educación. 12(12), Lima, UNMSM.

Vargas, A. (2004). Antes y después de las inteligencias múltiples. Educare. № 7 Recuperado de: http://www.revistas.una.ac.cr/index.php/EDUCARE/article/view/1121

Villamizar, G. (2011). Teorías implícitas de la inteligencia en el ámbito pedagógico. Psicogente, 14(26) 321-335 Diciembre. Universidad Simón Bolivar, Barranquilla, Colombia Recuperado de: http://portal.unisimonbolivar.edu.co:82/rdigital/ psicogente/index.php/psicogente

Walter, S., Schneider, M., Frega, J.y Carvalho de Souza, M. (2009). Similaridades e divergências no desenvolvimento das inteligências múltiplas de um Curso de Ciências Contábeis: um comparativo entre cursos, turmas e géneros. Revista Brasileira de Gestão de Negócios, Sao Paulo, 11(31) 134-151, abr/jun

\section{AGRADECIMIENTO}

Un reconocimiento especial al Centro de Investigación de la Universidad Ricardo Palma (CIURP), al Vicerrectorado de Investigación y al Rectorado de la Universidad Ricardo Palma por el apoyo administrativo y el financiamiento del trabajo de investigación. Así mismo a los Decanos, al Director del Programa de Estudios Básicos PEB que autorizaron la aplicación de los instrumentos $y$, a los alumnos que contestaron los mismos de forma desinteresada. Igualmente un agradecimiento especial a los docentes de aulas que permitieron la aplicación de los tests. 